\title{
Serum trypsin and TCR as novel markers for predicting disease activity in IgG4-related disease
}

\author{
WANG FENGQING ${ }^{l}$, YI QIANG ${ }^{l}$, GAO FENG ${ }^{2}$, ZHUANG ZEHAO ${ }^{3}$, MA YUEFEI $^{l}$, CHEN JING ${ }^{l}$, \\ WANG GUINA ${ }^{4}, H U$ BING ${ }^{4}, Z H E N G ~ J I N G^{4}, Z H A N G ~ J I N G J I N G^{4}, L U$ DANFENG ${ }^{4}, H_{A}$ RUI ${ }^{4}$, \\ LIU QICAI
}

'Department of Laboratory Medicine, the First Affiliated Hospital, Fujian Medical University, Fuzhou, Fujian Province, PR China ${ }^{2}$ Department of Pathology, the First Affiliated Hospital, Fujian Medical University, Fuzhou, Fujian Province, PR China ${ }^{3}$ Department of Gastroenterology, the First Affiliated Hospital, Fujian Medical University, Fuzhou, Fujian Province, PR China ${ }^{4}$ University Students' innovative team of Fujian Medical University, Fujian Medical University, Fuzhou, Fujian Province, PR China

\begin{abstract}
Background: IgG4-related disease (IgG4-RD) is a novel disease named in recent years. Because of its varied clinical manifestations, like tumor but not tumor, it brings a great challenge to clinical diagnosis. Trypsin and T-cell receptor (TCR) are thought to mediate the regulation of $B$ cell maturation, survival and antibody production. In this study, we investigated the clinical features and important novel markers of IgG4-RD.

Material and methods: A prospective cohort study of 22 patients with IgG4-RD was carried out from May 2009 to December 2012, and 65 cases with acute pancreatitis, 60 cases with pancreatic cancer and 120 healthy individuals were studied as controls. Serum TCR, trypsin and IgG4 levels were measured during pre- and post-treatment in the patients with IgG4-RD and their correlations with IgG4 were also assessed.

Results: Serum IgG4 and IgE levels in all patients were significantly increased, and tumor markers (carbohydrate antigen 19-9 and/or carbohydrate antigen 125) were also increased (12/22). Serum trypsin in patients with IgG4-RD was lower than in the ones with acute pancreatitis, pancreatic cancer, and healthy individuals. But serum TCR of IgG4-RD was significantly higher than in the pancreatic cancer group and normal controls and it was inversely correlated with the levels of IgG4 $(r=-3.160$, $p=0.042$ ).

Conclusions: The results indicate that serum TCR and trypsin might be useful markers for predicting disease activity in IgG4-RD.
\end{abstract}

Key words: IgG4-RD, serum IgG4, trypsin, TCR.

(Centr Eur J Immunol 2014; 39 (2): 193-197)

\section{Introduction}

Elevated serum IgG4 is the most remarkable feature of IgG4-related disease (IgG4-RD), and a large number of lymphocytes and IgG4 positive plasma cells can be seen in the involved tissue. The clinical symptoms are different for different organs involved. There may be blocked, compression symptoms or organ atrophy, and there may also be a result of cell infiltration or fibrosis resulting in organ failure [1-5]. Therefore, IgG4-RD includes a wide variety of diseases, including autoimmune pancreatitis, Mikulicz's disease, hypophysitis, Riedel thyroiditis, interstitial pneumonitis, interstitial nephritis, prostatitis, lymphadenopathy, retroperitoneal fibrosis, inflammatory aortic aneurysm, and inflammatory pseudotumor [6, 7]. Trypsin and T-cell receptor (TCR) are thought to mediate the regulation of $\mathrm{B}$ cell maturation, survival and antibody production $[8,9]$. The aim of this study was to investigate the contribution of serum trypsin and TCR in the pathogenesis of IgG4-RD.

\section{Material and methods}

\section{Patients}

This study was approved by the Fujian Medical University Committee and all study participants gave informed consent to serum analysis. Clinical information for the survey purposes was obtained by personal interviews using

Correspondence: Qi-Cai Liu, Department of Laboratory Medicine, the First Affiliated Hospital, Fujian Medical University, 20 Chazhong Road, Fuzhou 350005, Fujian Province, China, e-mail: lqc673673673@163.com 
a structured questionnaire and/or clinical trials. From May 2009 to December 2012, 22 unrelated IgG4-RD patients were studied. The patients were diagnosed on the basis of clinical pathological findings [3, 4], clinical findings (diffuse/focal enlargement or mass formation, nodular/thickened lesions in one or more organs), elevated serum IgG4 concentration (>135 mg/dl), and histopathologic features including infiltration of lymphocytes and IgG4-positive (+) plasma cells $(\mathrm{IgG} 4+$ plasma cells/IgG+ plasma cells $>40 \%$ and/or IgG4+ plasma cells $>10$ cells in 5 high-power fields) with typical tissue fibrosis or sclerosis. At the same time, 65 cases with acute pancreatitis, 60 cases with pancreatic cancer and 120 normal controls collected in the past four years were studied. Serum samples were obtained from the patients with IgG4-RD before and after glucocorticoid treatment.

\section{Clinical features}

Serum TCR (TCR $\alpha$ and TCR $\beta)$ and trypsin levels were determined using an enzyme-linked immunosorbent assay (ELISA) kit (Boyan, NingBo, China) pre- and post-glucocorticoid therapy in patients with IgG4-RD, pancreatic cancer, acute pancreatitis, and normal controls. Detection of serum IgG and IgG4 was measured by latex-enhanced nephelometric immunoassay (Dade Behring Marburg $\mathrm{GmbH}$, Germany).

\section{Histology and immunostaining}

Pancreas and other specimens of IgG4-RD were analyzed. The specimens were stained with hematoxylin and eosin (H\&E). Immunostaining for infiltrating plasma cells was performed using monoclonal antibody against human IgG4 (Zymed Laboratory, San Francisco, CA, USA, or the Binding Site, Birmingham, UK), antihuman IgG (Dako, Glostrup, Denmark), and/or antihuman CD138 (AbD Serotec, Oxford, UK). IgG4+ plasma cells were counted in five different high-power fields (HPF) with intensive infiltration, and the average IgG4+ plasma cell count was calculated. Average of $\mathrm{IgG} 4+/ \mathrm{IgG}+$ or $\mathrm{IgG} 4+/ \mathrm{CD} 138+$ plasma cell ratio of at least two different HPF (2-5 HPF) were calculated.

\section{Statistical analysis}

All data were analyzed with SPSS version 18.0 (SPSS Inc., Chicago, IL, USA). Mann-Whitney U test or Fisher's exact probability test was employed for the statistical analyses. A $p$ value of $<0.05$ was considered statistically significant. Data are shown as mean \pm standard deviation (SD).

\section{Results}

\section{Clinical and laboratory features of IgG4-RD}

The mean serum IgG level was $10.46 \mathrm{~g} / \mathrm{l}$ (range 5.12$36.00 \mathrm{~g} / \mathrm{l})$, and the mean serum IgG4 level was $6.501 \mathrm{~g} / \mathrm{l}$ (range 2.110-16.400 g/l) (Table 1). All of the patients had elevated serum IgG4 and IgE. C-reactive protein (CRP) (16/22), carbohydrate antigen 19-9(6/22), carbohydrate antigen 125 (12/22) were increased, but antinuclear antibody and anti-extractable nuclear antigen (ENA) were negative. The most frequent clinical manifestations were painless obstructive jaundice (17/22) and weight loss (16/22) in our cohort.
A

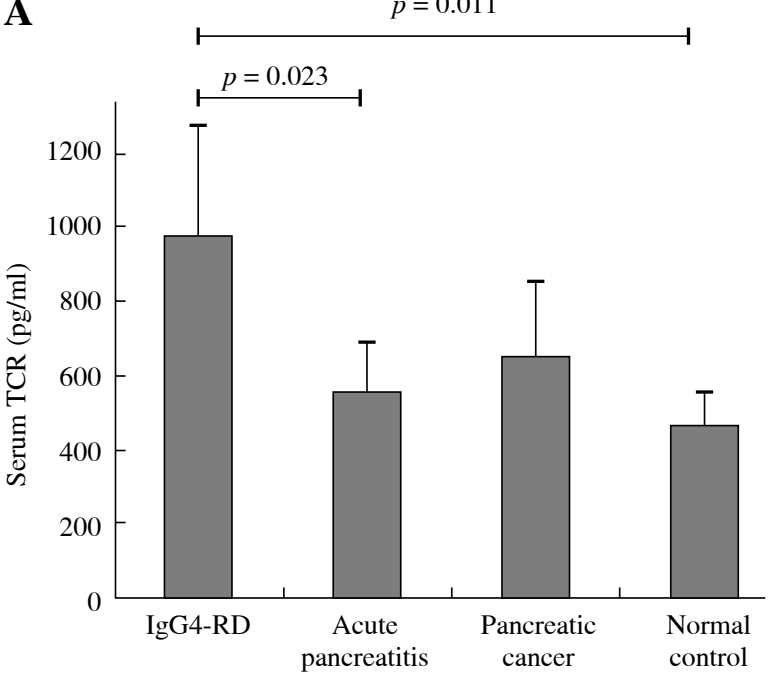

B

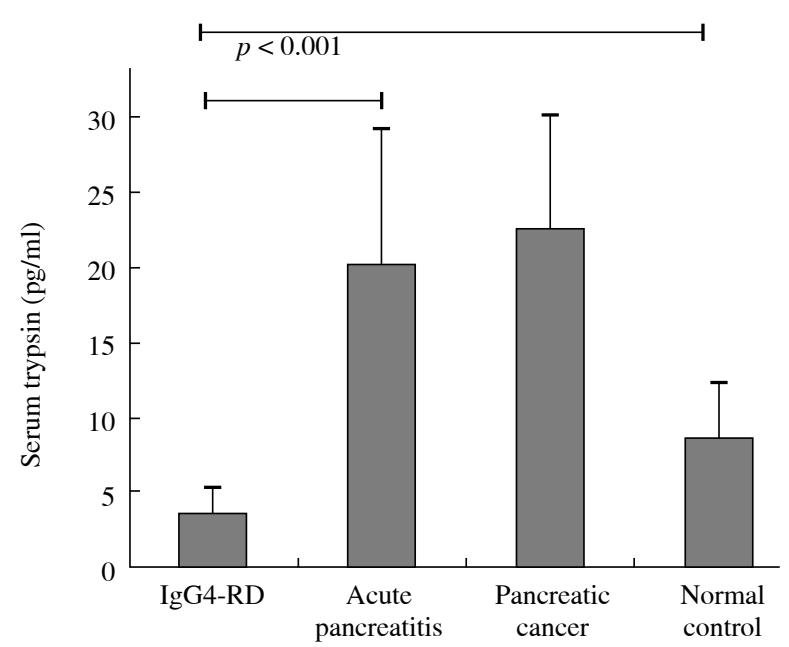

Fig. 1. Serum TCR and trypsin in IgG4-RD, acute pancreatitis, pancreatic cancer, and normal controls. A) Serum levels of TCR in patients with IgG4-RD, acute pancreatitis, pancreatic cancer, and normal controls. B) Serum levels of trypsin in patients with IgG4-RD, acute pancreatitis, pancreatic cancer, and normal controls 


\section{Serum TCR increased and trypsin decreased in IgG4-RD}

Serum TCR in patients with IgG4-RD $(n=22,968.7$ $\pm 393.2 \mathrm{pg} / \mathrm{ml}$ ) was significantly higher than in healthy controls $(n=120,556.9 \pm 163.6 \mathrm{pg} / \mathrm{ml})$, acute pancreatitis $(n=65,652.8 \pm 322.5 \mathrm{pg} / \mathrm{ml})$, and pancreatic cancer $(n=60,467.9 \pm 211.9 \mathrm{pg} / \mathrm{ml})(p<0.05)$ (Fig. 1A). And there were no significant differences in IgG4-RD pre- and post-treatment $(p=0.383)$, while serum trypsin of IgG4$\mathrm{RD}(3.3 \pm 0.8 \mathrm{ng} / \mathrm{ml})$ was lower than in acute pancreatitis $(24.2 \pm 8.2 \mathrm{ng} / \mathrm{ml})$, pancreatic cancer $(22.6 \pm 10.5 \mathrm{ng} / \mathrm{ml})$, and normal controls $(8.5 \pm 3.2 \mathrm{ng} / \mathrm{ml})(p<0.05)$ (Fig. 1B).

\section{Relationship between serum TCR and trypsin and IgG4}

Of the 22 patients analyzed in this study, serum TCR was significantly associated with $\mathrm{IgG} 4$ during the whole investigation period [serum TCR $(\mathrm{pg} / \mathrm{ml})=-3.1602 \mathrm{IgG} 4$

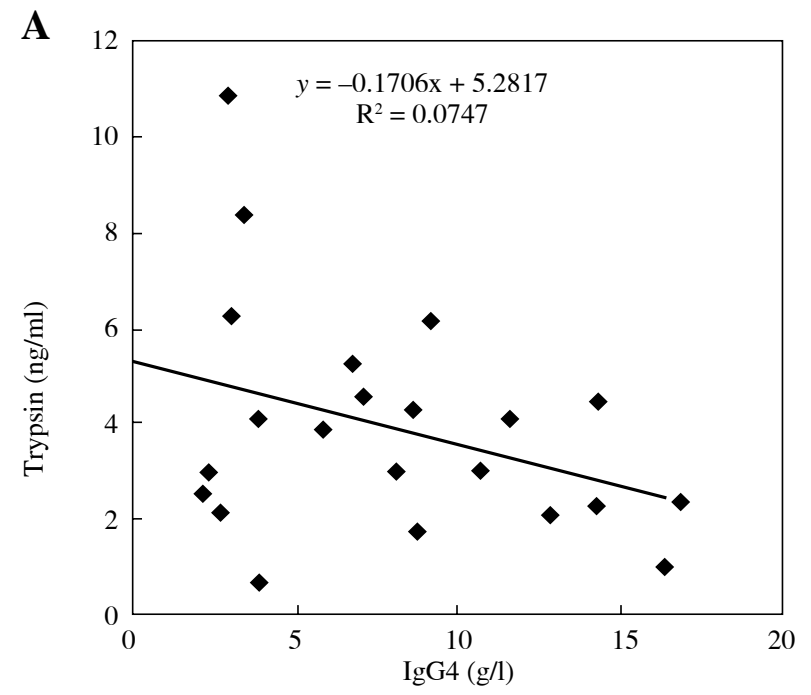

$(\mathrm{g} / \mathrm{l})+594.78, \mathrm{R}^{2}=0.042$ ] (Fig. 2A). The same applied to trypsin and $\mathrm{IgG} 4$ in patients with IgG4-RD [trypsin (ng/ $\mathrm{ml})=-0.1706 \mathrm{IgG} 4(\mathrm{~g} / \mathrm{l})+5.2817, \mathrm{R}^{2}=0.0747$ ], and serum trypsin concentration decrease with an increasing IgG4 concentration.

\section{Pathological examination}

As shown in Fig. 3, a large number of lymphocytes and plasma cells were found in the involved organs (Fig. 3B). IgG4 positive cells accounted for the proportion of IgG positive plasma cells $>40 \%$ (Fig. 3C).

\section{Discussion}

Kamisawa for the first time put forward the concept of IgG4-RD in 2003. After that, the disease gradually attracted extensive attention and recognition all over the world [6-11]. In 2010, a review titled "a new syndrome: clinical disease spectrum was related to IgG4" announced the uni-

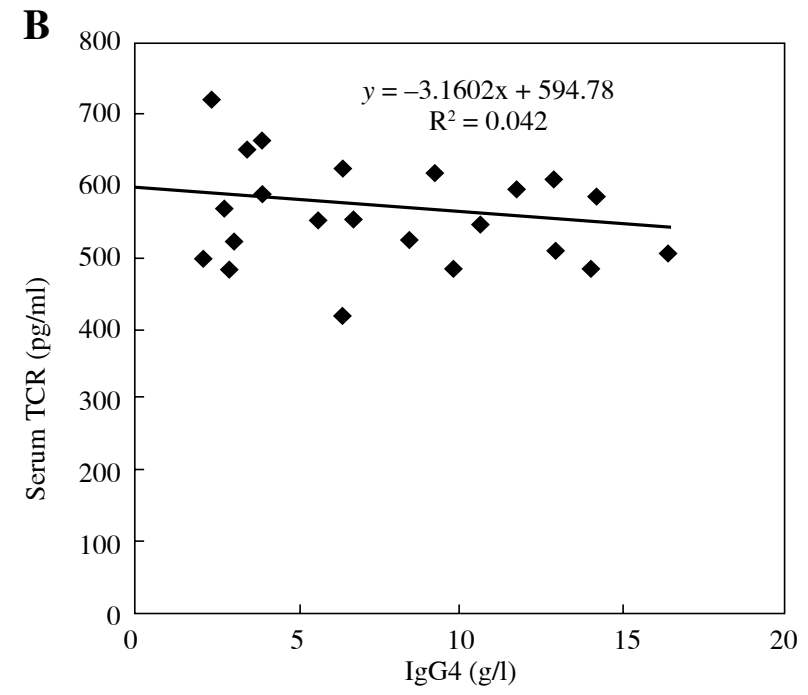

Fig. 2. Relationship between serum TCR and trypsin, and IgG4 in patients with IgG4-RD. A) Correlation between serum levels of trypsin and IgG4 in patients with IgG4-RD, the relevance is serum TCR (pg/ml) = -3.1602 IgG4 (g/l) +594.78, $\mathrm{R}^{2}=0.042$. B) Correlation between serum levels of TCR and IgG4 in patients with IgG4-RD, the relevance is trypsin (ng/ml) $=-0.1706 \mathrm{IgG} 4(\mathrm{~g} / \mathrm{l})+5.2817, \mathrm{R}^{2}=0.075$, and serum trypsin concentration decreases with an increasing IgG4 concentration
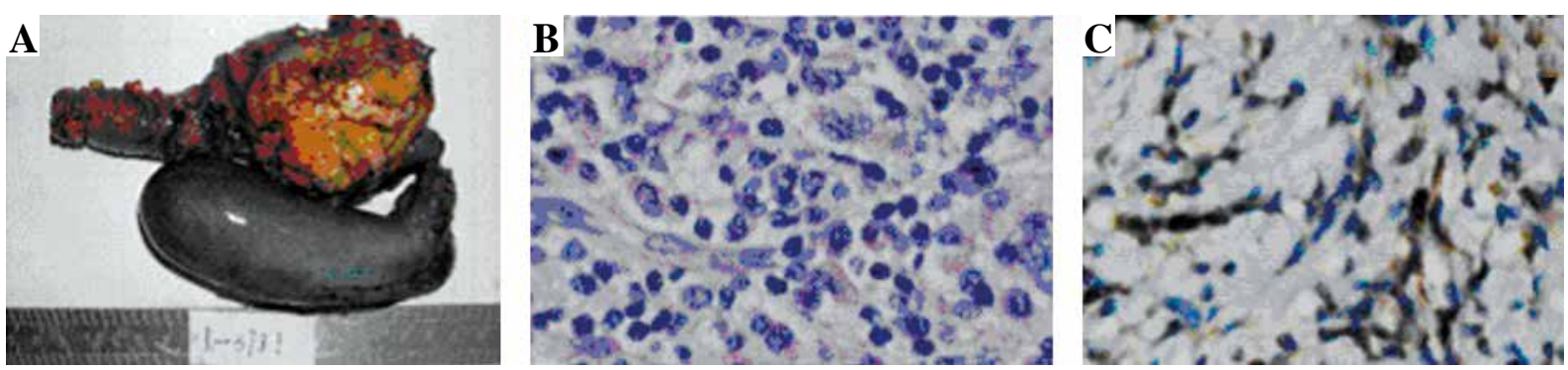

Fig. 3. Histopathologic examination of the involved organs. A) Gross sample. B) H\&E stain. C) Immunostaining for IgG4 or IgG, magnification $400 \times$ 
fied name [8]. In recent years, the Chinese began to pay attention to IgG4-RD and some cases of clinical research were reported [12].

The disease is a multisystem complex disease, with a more common onset in the elderly, the most frequent involved organs are the pancreas, liver, nervous system, lacrimal gland, and salivary gland. The involved organs were subject to hyperplasia enlargement due to fibrosis, chronic inflammation, which leads to the oppression of obstructive symptoms or dysfunction. It is not easy to make the diagnosis and differential diagnosis, but the disease generally improved after glucocorticoid treatment [11-15]. So far there has been no large-scale IgG4-RD epidemiological investigation and its incidence and prevalence are still unclear [1619]. In this study, the average age of patients was 48.8 years, the male to female ratio of $2.33: 1$, the important clinical manifestations are jaundice and weight loss, the common involved organs are the pancreas and bile duct, and the clinical symptoms were improved after hormone treatment.

Because the imaging and clinical features of IgG4-RD are extremely similar to tumor [20-23], most patients were treated by operation. Because its relative lack of clinical understanding of IgG4-RD and the IgG4-RD related auxiliary examination is rare, so misdiagnosis and missed diagnosis are common. The study found that serum IgG4 was significantly higher in patients with IgG4-RD, which seems to suggest that serum IgG4 can be used as diagnostic indicators of IgG4-RD and tumor. But the data also show that more than half of the patients with IgG4-RD have tumor markers increased and some cancers such as pancreatic cancer are also found to have serum IgG4 increased; it brings a great challenge to make a differential diagnosis. Recent studies have reported that IgG4-related disease sometimes involves the systemic lymph nodes and it is undeniable that TCR is active in the process of the autoimmune [24-25]. In this study, we investigated the changes of serum TCR pre- and post-treatment in patients with IgG4-RD. It has been found that the IgG4-RD group showed no significant changes, but it was significant higher than in pancreatic cancer, pancreatitis group and normal controls. What is more, trypsin can be used as a signal factor of lymphocyte stimulation. The study found that serum trypsin in patients with IgG4-RD significantly increased after treated. Therefore, serum IgG4, TCR and trypsin are expected to become the diagnosis markers of IgG4-RD.

Although IgG4-RD has been paid more and more attention, the exact etiology, pathophysiology and epidemiology still need to be further studied. As an interdisciplinary system disease, it requires multiple areas of medical mutual cooperation, and realizes the sharing of resources through the effective platform. Further longitudinal studies with larger numbers of patients are required to determine the role of trypsin/TCR system and to determine whether trypsin and TCR might serve as therapeutic targets in IgG4-RD.
The authors declare no conflict of interest.

Financially supported by the Natural Science Foundation of Fujian Province (2013J01302), National Natural Science Foundation of China (81201362, 81201590, and 21275028), Fujian Medical Innovations (2012-CXB-21), Foundation of Fujian Education Department (JA12143), Outstanding Youth Foundation of Fujian Provincial Higher Education (JA12133) and Fujian Provincial Education, No. JA12143.

\section{References}

1. Umehara H, Okazaki K, Masaki Y, et al. (2012): A novel clinical entity, IgG4-related disease (IgG4RD): general concept and details. Mod Rheumatol 22: 1-14.

2. Stone JH, Zen Y, Deshpande V (2012): IgG4-related disease. N Engl J Med 366: 539-551.

3. Okazaki K, Uchida K, Koyabu M, et al. (2011): Recent advances in the concept and dianosis of antoimmune pancreatitis and IgG4-related disease. J Gastroenterol 46: 277-288.

4. Carruthers MN, Stone JH, Khosroshahi A (2012): The letest on IgG4-RD: a rapidly emerging disease. Curr Opin Rheumatol 24: 60-69.

5. Divatia M, Kim SA, Ro JY (2012): IgG4-related sclerosing disease, an emerging entity: a review of a multi-system disease. Yonsei Med J 53: 15-34.

6. Gornowicz-Porowska J, Pietkiewicz P, Bowszyc-Dmochowska M, Dmochowski M (2013): Clinical immunology Immunoglobulin G4 is prevailing over immunoglobulin G1 in autoimmunity of pemphigus and bullous pemphigoid: analysis of tissue-bound antibodies in active diseases. Centr Eur J Immunol 38: 80-91.

7. Sato Y, Kojima M, Takata K, et al. (2009): Systemic IgG4-related lymphadenopathy: a clinical and pathologic comparison to multicentric Castleman's disease. Modern Pathology 22: 589-599.

8. Takahashi H, Yamamoto M, Suzuki C, et al. (2010): The birthday of a new syndrome: IgG4-related disease constitutes a clinical entity. Autoimmun Rev 9: 591-594.

9. He YD, Ma N, Xiao H, et al. (2013): B cell activating factor (BAFF) induces the transcription of recombination-activating genes in transitional stage $1 \mathrm{~B}$ cells. Centr Eur J Immunol 38: 336-342.

10. Hart PA, Moyer AM, Yi ES, et al. (2012): IgG4-related paratesticular pseudotumor in a patient with autoimmune pancreatitis and retroperitoneal fibrosis: an extrapancreatic manifestation of IgG4-related disease. Hum Pathol 43: 2084-2087.

11. Chari ST, Kloeppel G, Zhang L, et al. (2010): Autoimmune Pancreatitis International Cooperative Study Group (APICS). Histopathologic and clinical subtypes of autoimmune pancreatitis: the Honolulu consensus document. Pancreas 39: 549-554.

12. Gao F, Li YM, Wang CD, et al. (2014): Identification of a novel frame-shift mutation in PRSS1 gene in Han patients with autoimmune pancreatitis. Curr Molecular Med 14: 1-9.

13. Dong F, Chen QQ, Zhuang ZH, et al. (2014): Multiple gene mutations in the patients with type 2 autoimmune pancreatitis and its clinical features. Centr Eur J Immunol in press.

14. Kawa S, Ito T, Watanabe T, et al. (2012): The Utility of Serum IgG4 Concentrations as a Biomarker. Int J Rheumatol 2012: 198-214. 
15. Sato Y, Ohshima K, Ichimura K, et al. (2008): Ocular adnexal IgG4-related disease has uniform clinicopathology. Pathol Int 58: 465-470.

16. Umehara H, Okazaki K, Masaki Y, et al. (2012): A novel clinical entity, IgG4-related disease(IgG4-Rd): general concept and details. Mod Rheumatol 22:1-24.

17. Umehara H, Okazaki K, Masaki Y, Kawano M, Yamamoto $\mathrm{M}$, Saeki $\mathrm{T}$, et al. Comprehensive diagnostic criteria for IgG4-related disease. Mod Rheumatol 2012; 22: 21-30.

18. Nishimori I, Tamakoshi A, Otsukin M (2007): Prevalence of autoimmune pancreatitis in Japan from a nationwide survey in 2002. J Gastroenterol 42: S6-S8.

19. Raina A, Yadav D, Krasinskas AM, et al. (2009): Evaluation and management of autoimmune pancreatitis: experience at a large US center. Am J Gastroenterol 104: 2295-2306.

20. Kamisawa T, Shimosegawa T, Okazaki K, et al. (2009): Standard steroid treatment for autoimmune pancreatitis. Gut 58 : 1504-1507.

21. Stone JH, Zen Y, Deshpande V (2012): IgG4-related disease. N Engl J Med 366: 1642-1647.

22. Kamisawa T, Egawa N, Nakajima H, et al. (2003): Clinical difficulties in the differentiation of autoimmune pancreatitis and pancreatic carcinoma. Am J Gastroenterol 98: 2694-2699.

23. Gao F, Li YM, Wang CD, et al. (2014): Identification of a novel frame-shift mutation in PRSS1 gene in han patients with autoimmune pancreatitis. Curr Mol Med 14: 340-348.

24. Yamada K, Kawano M, Inoue R, et al. (2008): Clonal relationship between infiltrating immunoglobulin G4 (IgG4)-positive plasma cells in lacrimal glands and circulating IgG4-positive lymphocytes in Mikulicz's disease. Clin Exp Immunol 152: 432-439.

25. Sugumar A, Levy MJ, Kamisawa T, et al. (2011): Endoscopic retrograde pancreatography criteria to diagnosis autoimmune pancreatitis: an international multicentric study. Gut 60: 666-670. 